\title{
CLINICAL EFFECTS OF TETRYL (CE POWDER)*
}

\author{
BY \\ H. B. TROUP \\ From the Royal Ordnance Factory, Bridgend
}

These observations are compiled from the findings and opinions of all the medical officers at R.O.F. Bridgend. No claim is made that the ideas are original; in fact, much of the material is common knowledge in filling factories, but tetryl has been used to a larger extent in this than in any other filling factory and it is thought that opinions based on this wide experience, whether new or not, should be recorded.

The problem at R.O.F. Bridgend has been all the greater on account of: (1) The large number of workers in contact with CE, approximately 5000 . (2) The wide distribution of $\mathrm{CE}$ throughout the factory; of 13 sections, 6 were always CE sections, 4 more had CE shops often on the section, and only 3 were entirely free from CE contact. (3) The very large labour turnover in the factory during the past two years. (4) The large intake of ageing men in this period.

CE powder has been the main cause of loss of work in this factory, chiefly owing to dermatitis which it produces. Some workers complain of respiratory and gastric trouble, but these conditions are of minor importance in comparison with the skin affections.

The type of rash differs in individuals and in the areas of the skin affected. The clinical types are as follows:

(1) Erythema. Erythema affecting the face and neck; this may look exactly like a mild sunburn. In a fair number of cases this clears up and gives no further trouble after treatment.

(2) As in (1) but the rash becomes very dry and scaly. These cases are much more likely to recur when put into contact again.

(3) As in (1) and (2) plus slight or very marked oedema of the eyelids. These cases are never able to go into contact again; in fact, many are so sensitive, that even a visit to the works surgery (outside the contact area) will produce a recurrence, and it may be necessary to visit them at home.

(4) As in (1), (2) and (3) plus conjunctivitis. These cases are never able to go into contact again.

(5) A more papular patchy type of rash seen on the neck, shoulders and forearms with a tendency to lichenefication.

* Tri-nitro-phenyl-methyl-nitramine. The contraction CE stands for 'compound, exploding,' and is used throughout the paper as a synonym for tetryl.
(6) Sensitisation. When a patient has become really sensitised a rash is liable to appear at any time on any area of the skin, usually face, back of neck, forehead, behind the ears, on the wrists. This has no relationship to his particular occupation and appears no matter where, and on what job, he works. In some cases it spreads generally on to the trunk and legs. Even if there is no rash on the body there is frequently marked irritation of the perineum.

(7) All the 6 types mentioned above vary from extreme mildness to extreme severity.

\section{Treatment}

It is seldom any good treating these cases whem they are still in contact; they are much better right away from the powder and all irritants, because when the skin becomes sensitive to $\mathrm{CE}$ it also seems to react more easily to other irritants even although they may be non-contact substances.

The applications used vary with the individual but generally lot. calamin. oleosa is best for the face, and ung. calamin. et boric. for the coarser rash on the neck, shoulders and wrists; on the other hand, some prefer the ointment on the face and others say that it irritates and prefer ung. sed. Those patients who complain of a burning feeling rather than an irritation get more relief from liq. alumin. acetat (B.P.C.); if this is too drying it can be alternated daily with lot. calamin. oleosa with good results. If there is a weeping area, which often occurs on the back of the neck and behind the ears, siccolam 3 parts and lanolin 1 part is very satisfactory. Treatment to a great extent is individual and symptomatic and therefore follows ordinary dermatological procedure.

\section{Cause of the Rash}

It is quite clear that personal idiosyncrasy to tetryl is the primary cause of the rash; all other causes or so-called causes are merely secondary or, one might say, provocative. Such secondary causes are:

(a) Lack of personal cleanliness. The worker may be dirty by nature. She may not make use of ablution rooms through laziness or because she wants to get off contact in order to get away from someone she does not like, the over-looker for example, or to join friends who have already been taken out of contact. These are not uncommon reasons; in fact it is a frequent experience to find. 
that a batch of girls will for no apparent reason produce a rash and they will try to stick together wherever they go.

(b) Careless use of barrier substances. Used carelessly through laziness, or for reasons as stated in $(a)$.

(c) General health. The worker's general condition, mental and physical, will affect susceptibility. Innumerable instances of this can be given. A particular instance is the emotional factor; this seems to have been the precipitating cause in a young married woman who had worked in CE for three years without developing a rash, but developed an acute $\mathrm{CE}$ dermatitis very soon after hearing that her husband was missing after air operations.

(d) Sex, hair colour and skin lesions. It is probable that some commonly accepted rules of selection require revising. Our experience is that $\mathrm{CE}$ rash is more frequent and far more severe in the male than in the female. Probable reasons for this are that the male skin with its hair is rougher and more likely to collect and hold powder; shaving removes the top layer of horny scale cells and exposes the more sensitive cells beneath to come into contact with the powder; in a woman the make-up (which most of them use) acts as a barrier; CE has no particular preference for reds, blondes or brunettes.

Skin lesions need not necessarily be regarded as a bar to CE contact; acne vulgaris, extensive scarring following burns, and pigmentation changes do not increase susceptibility and do not add to the gravity of a rash after it is acquired. Even psoriasis has been exposed with impunity to $\mathrm{CE}$ over long periods, but seborrhoea certainly, and most other skin diseases, are regarded as factors which must exclude from CE contact; so also is a dry scaly skin, the type of skin which is labelled ichthyosis in its more obvious form, for here a most intractable CE dermatitis can arise.

It is probable that in view of what is to be said later of the systemic effects of $\mathrm{CE}$, selection should take greater cognizance of alimentary disorders than has been the custom.

\section{Effect of Environment}

The distribution of the rash and the severity depends to a certain extent on the type of work being done. Work with CE may include putting pellets into paper cartons which tends to spray the powder on to the face (see figs. 1 and 2). Hand stemming is more likely to affect the hands or the wrists. The general cleanliness of the shop has an effect on all this which can be shown by testing the air for powder concentration * and comparing the rash incidences.

It seems that the incidence of rash is higher in winter than in summer. This is contrary to earlier conceptions that rash was more prevalent in hot weather, but it is probably explained by the fact that shop doors are kept wide open in the summer with consequent better ventilation.

\footnotetext{
* The maximum allowable atmosphere concentration of tetryl (CE) is $1.5 \mathrm{mg} . / \mathrm{m}^{3}$ It is believed that there is no health hazard at this concentration. (Manual of Industrial Hygiene (1943). Ed. by W. M. Gafafer. W. B. Saunders Company, p. 264.)
}

The incidence rate on the Pellets Section (see figs. 1 and 2) is three times as high as it is in the rest of the factory, but correlation between incidence rate and concentration of airborne $\mathrm{CE}$ is distorted by the impingement of other factors. For instance, the incidence is consistently highest in the Wrapping Shops, though the air concentration of CE has been six times higher in the Press'Shops and three times higher in Bag Stemming Shops than in the Wrapping Shops. This is probably due to the fact that newcomers to the Pellets Section and those returning to contact, i.e. the 'susceptibles' were allocated to the Wrapping Shops. So, also, incidence was high in the Pool Shop where CE air concentration was low, but irritant solvents (naphtha and spirit) provided an additional hazard. Recurrence rate was cut when those returning to contact were no longer drafted direct into the Pool Shop.

\section{The Eyes}

CE conjunctivitis may be due to gross contamination (rubbing the eye) but is more commonly due to airborne CE. It is usually, though not invariably, accompanied by a CE rash of the face and will often persist for a long period after the rash has subsided. Irido-cyclitis and keratitis have occurred as a direct sequel of $\mathrm{CE}$ conjunctivitis. Sensitization may be extreme in conjunctivitis and in some cases a visit to the factory surgery has sufficed to give rise to an acute relapse.

\section{Respiratory Tract}

The respiratory effects of $\mathrm{CE}$ are difficult to assess but it is accepted that tracheitis and asthma do result from exposure to $\mathrm{CE}$. The ex-miner who has been certified as suffering from silicosis or pneumokoniosis is excluded from CE contact, but the lesser disability or reticulation has proved to be no bar to work in contact with $\mathrm{CE}$.

\section{Alimentary Tract}

The main symptoms of gastric disorder due to $\mathrm{CE}$ are nausea and anorexia, pain being a less common and later symptom than in the case of TNT gastritis. It is thought that although gastric disorders are less obvious with $\mathrm{CE}$ contact than in TNT contact, gastric symptoms are, in fact, not uncommon. That this should be so might be expected from the similarity in molecular structure of CE and TNT.

In this connection we had two cases of jaundice in which the toxic effect of $\mathrm{CE}$ though not proven cannot be entirely eliminated.

Case 1. M. W. Age 24; in contact with CE from 18.11.41 until 28.9.43, when she went sick. She was out of sorts since September 1943, complaining of nausea and epigastric pain, which became worse on 26.9.43, and jaundice was noticed on 29.9.43. She was admitted to hospital for investigation on 4.10.43, and it was reported that the urine contained an excess of urobiligen, urobilin and bile pigment and that the Van den Burg gave a negative direct and a positive indirect reaction; the opinion expressed was that it was difficult to disprove the possibility of a toxic factor in causing the jaundice. 


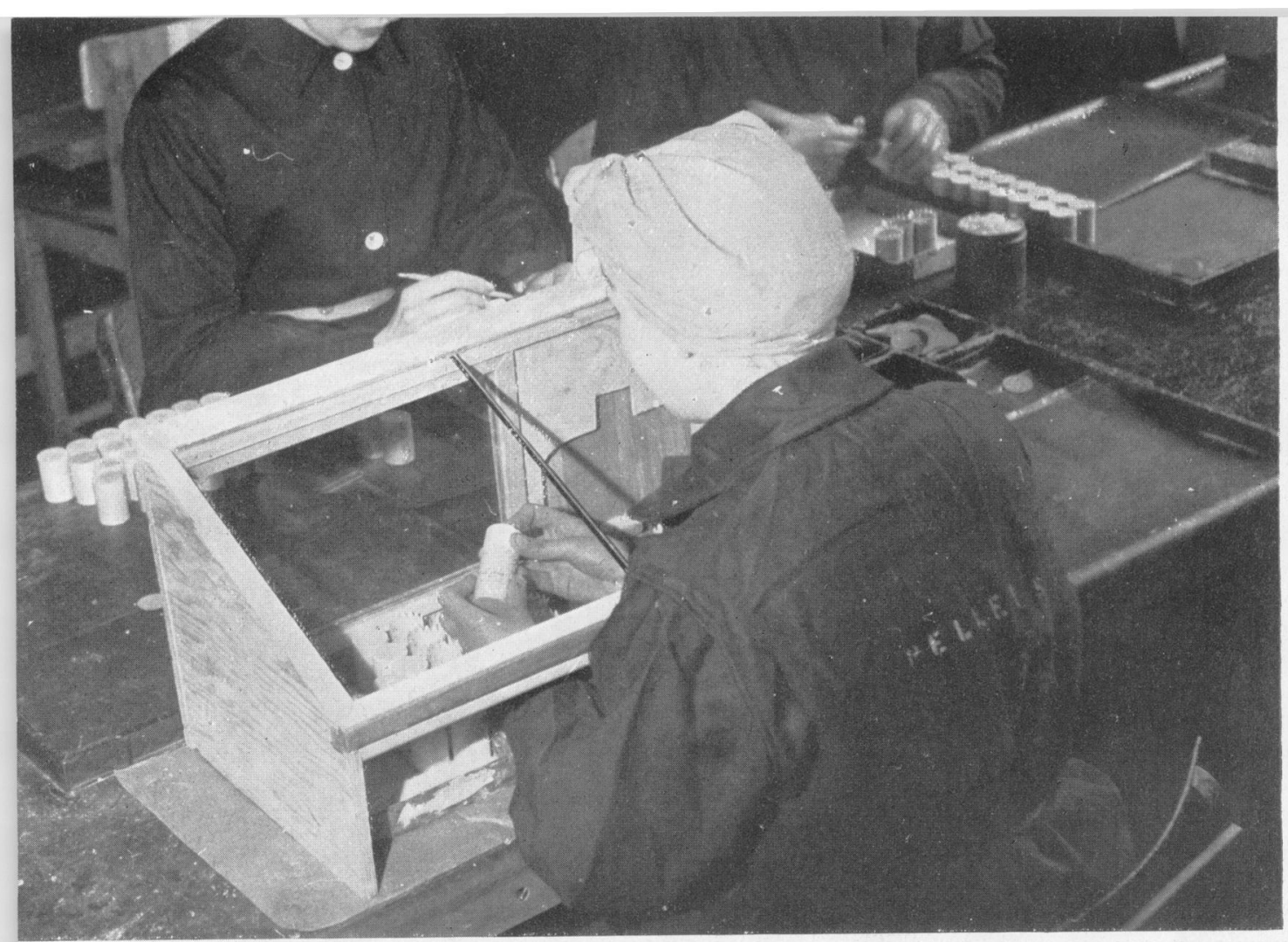

FIG. 1 (above).--Inserting pellets of tetryl (CE powder) into cartons. Note the protection against dust provided by fixed glass screen - the 'lighthouse.'

Fig. 2 (below).-Team packing cartons of tetryl. Another view of the ' lighthouse' showing its protective value against tetryl dust.

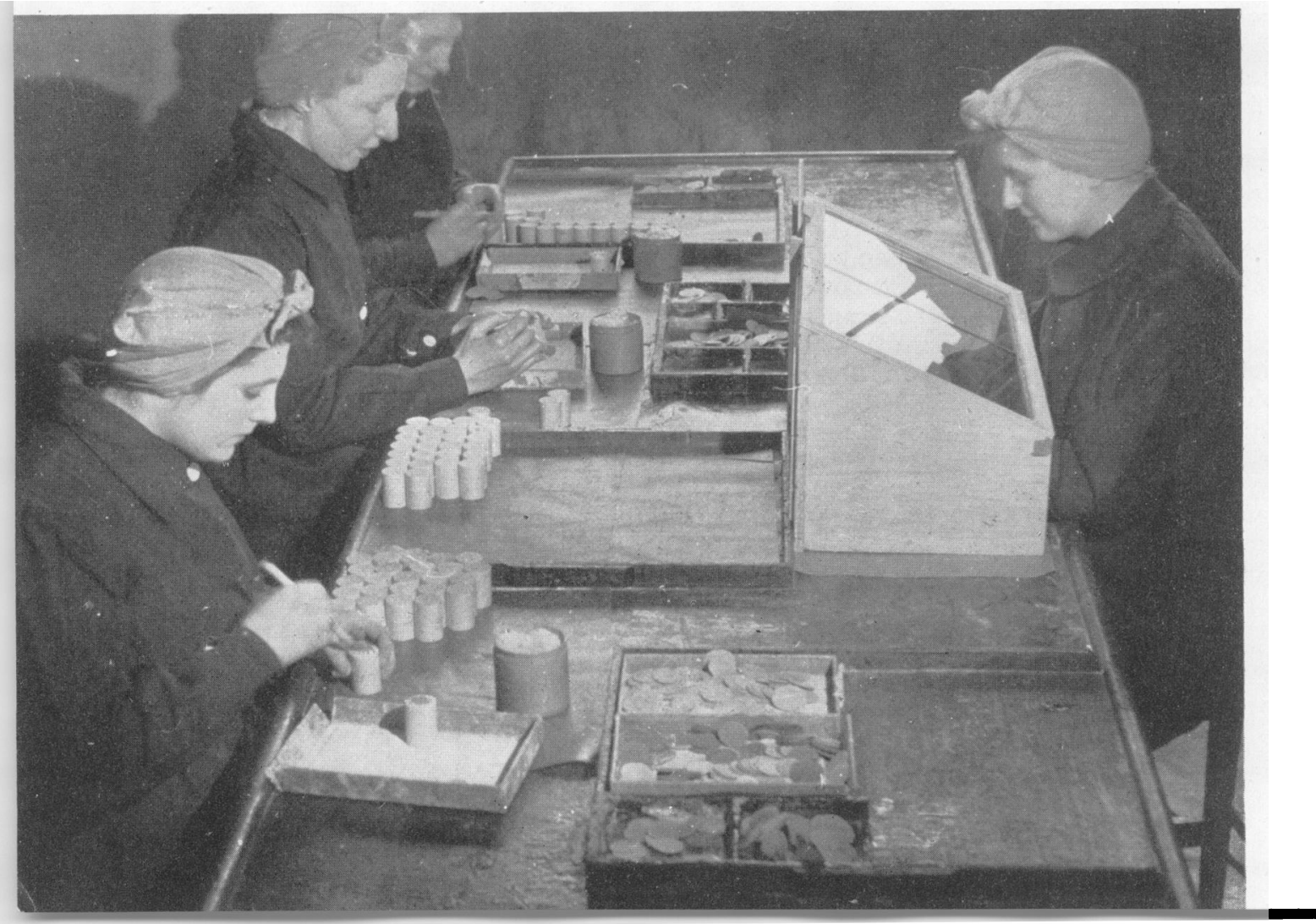


Case 2. B. D. Age 22; worked in the factory from 9.6.41 until 24.5.43; in contact with CE much of the time but never in contact with TNT. She died following clinical symptoms of toxic jaundice and the post-mortem findings were atrophy of the liver with distortion of the upper parts of the liver. After leaving the R.O.F. she had worked at a factory where she handled closed containers which had been filled with chlorinated naphthalene.

\section{Prophylaxis}

The main factor in prevention is personal cleanliness and shop cleanliness. Prevention can be attained by the following measures.

\section{(1) Personal}

All workers in CE Sections are given the factory booklet which sets out the objects of personal prophylaxis and the means by which it is carried out.

(a) Barrier substances. The most satisfactory are the varnishes, particularly Manucol, a sodium alginate modified for our requirements, and selected as a barrier on CE Sections; it was chosen because it forms a quick-drying cohesive pellicle on the skin surface. Sulphite soap is also provided, in tablet form, which is effective and more readily accepted than liquid soap by the workers. Whichever barrier is used the application requires instruction and supervision. It is applied before starting work and again in the break. The application before the break should be washed off at the break and reapplied before work is again commenced. This should be washed off at the end of the shift. Clean towels should be used at each washing and a special receptacle kept for the dirty towels. Creams are not considered to be very satisfactory as they tend to collect the powder.

(b) Hair protection. This is most desirable but seems impossible to achieve. The various headdresses last for a day or two but the female instinct for adornment and attraction prevails and 'the curls will out.' Hair protection is of considerable importance because if a person sensitized to $C E$ is living in the same house, the powder carried home in the hair will produce a rash in that person. This has occurred on a number of occasions here and the only thing to do is to take the whole household out of contact; as there may be several persons living in the house who are working on contact this may have a considerable effect on production.

In order to establish routine measures it has been made the responsibility of the factory nurses to supervise personal prophylaxis of those who are in the Training School and also of those who have returned to contact. Other operatives are the responsibility of the production staff, who must be left in no doubt of this responsibility.

\section{(2) In the Shops}

$\mathrm{CE}$ rash is much more commonly due to airborne $\mathrm{CE}$ than to direct contamination and no great progress will be made in reducing the incidence of $\mathrm{CE}$ rash until extract ventilation is installed at the site of the operations, e.g. gridded tables with down draught. The problem of extract ventilation is complicated by the explosive risk but it can be carried out safely by induced draught and, however great the difficulties, it should be installed in those factories where there are widespread CE processes.

The following measures were carried out in this factory with the object of minimizing the incidence of rash:

(a) The spread of CE operations on to so many sections made it impossible to remove effectively from contact those who were very sensitive to $\mathrm{CE}$ and in a number of cases removal from contact did not prevent progressive deterioration and eventual permanent unfitness for contact. To overcome this difficulty a special shop was opened on a part of one of the remote non-contact sections and transfer to and from this shop was effected on the recommendation of the medical officer if he considered the worker to be too sensitive to be dealt with in the available non-contact sections. Clean, light jobs were done in this shop and the majority, though not all of those who worked there, were reconditioned at least to work on non-contact sections. It is important that a filling factory in which $C E$ is widespread must have sufficient sections which are strictly isolated from CE Sections, i.e. without common cleanways.

(b) A sub-committee of the factory safety committee did excellent work and helped to instil a sense of urgency in the production management.

(c) Shop ventilation in winter months was improved by the provision of plywood inclined baffles inside the louvres, whereby incoming air was directed upwards and operatives no longer needed to stuff up the louvres.

(d) Squads of cleaners were detailed to the sole and constant duty of cleaning the CE presses.

(e) The use of a specially designed ' lighthouse' (see figs. 1 and 2) was made compulsory during the insertion of pellets into cartons; this certainly reduced the airborne $C E$ which had been previously puffed up into the face of the operator.

$(f)$ Later the 'lighthouses' were dispersed as widely as possible in the Wrapping Shops and operatives worked as isolated small teams instead of being bunched along a table.

\section{Summary}

Tetryl (known colloquially in Royal Ordnance Factories as CE) is a potent cause of dermatitis. This occurs mainly as erythema of the face.

Clinical effects on other parts of the body are insignificant.

Personal idiosyncrasy is the main causal factor. Contributory or secondary factors are lack of personal cleanliness, careless use of barrier substances, poor general health.

The rash is commoner and more severe in men.

Skin lesions of any kind are a bar to contact with this substance.

Prevention is not difficult and consists of personal and shop cleanliness. Health propaganda, barrier substances, hair protection and adequate shop ventilation and guarding by 'lighthouses' are important measures. 